\title{
scripted
}

Volume 14, Issue 1, June 2017

\section{Adaptive Pathways Regulations for Stem Cells: Accelerating Access to Medicine or Deregulating Access to Markets?}

\author{
Tsung-Ling Lee and Tamra Lysaght**
}

\author{
(ㄷ) (1) $(9)$ \\ (C) 2017 Tsung-Ling Lee and Tamra Lysaght \\ Licensed under a Creative Commons Attribution-NonCommercial- \\ NoDerivatives 4.0 International (CC BY-NC-ND 4.0) license
}

DOI: $10.2966 /$ scrip.140117.81

\begin{abstract}
In response to calls for faster access to innovative biomedicines, the European Medical Agency ran a two-year pilot program known as the "Adaptive Pathways" (AP) scheme. Under this approach, evidence is gathered and evaluated iteratively for license adaptation to reduce uncertainties rather than in an accumulative phased trial process. With the European Medicines Agency (EMA) aiming to mainstream the AP scheme, the regulatory approach of accelerating the approval process for novel biomedicines is in need of critical evaluation and should be viewed with some caution. Focusing on the recent market authorisation of a stem cell product, we scrutinise the legal and ethical merits of this programme. We draw attention to how the AP scheme will grant conditional marketing approval to medicinal products with limited clinical benefits. In response
\end{abstract}


to the identified weaknesses of the AP scheme, we propose procedural safeguards which are in keeping with the EMA's public health missions.

\section{Keywords}

adaptive pathways; stem cell regulations; adaptive licensing; conditional marketing approval; Holoclar; EMA

* Research Fellow, Centre for Biomedical Ethics, National University of Singapore, Singapore. Email: t1265@georgetown.edu

** Assistant Professor, Centre for Biomedical Ethics, National University of Singapore, Singapore 


\section{Introduction}

Stem cells and cell-based products have the potential to generate new and innovative treatment modalities. Yet, the nature and characteristics of these products present novel and complex challenges for regulators. As a result, very few stem cell-based products have received regulatory approval either in the European Union (EU) or other major markets, such as the United States (US). ${ }^{1}$

Critics of the current regulatory regime argue that conventional approval processes are hampering innovation with rigid and inefficient methodologies that are unable to respond adequately to continuously emerging knowledge and uncertainties inherent to novel biomedicines. ${ }^{2}$ In response, the European Medicine Agency (EMA) ran a pilot project between March 2014 and August 2016 to trial an adaptive licensing scheme, now referred to as "adaptive pathways" $(\mathrm{AP})$.

In this paper, we focus on the EU's AP scheme for the accelerated market approval of stem cell-based production, highlighting two potentially problematic issues: the use of surrogate endpoints, and the lack of robust regulatory oversight in post-marketing approval phase. To illustrate our argument, we reference Holoclar, the first stem cell-based product the EMA has granted market authorisation under the AP scheme, as a case study. While we recognise the imperative of exploring alternative pathways for stem cell-based products, we

1 See e.g. EU Press release, "First Stem-Cell Therapy Recommended for Approval in EU" (2014), available at

http://www.ema.europa.eu/ema/index.jsp?curl=pages/news and events/news/2014/12/news detail 002239.jsp\&mid=WC0b01ac058004d5c1 (accessed 3 April 2017).

2 EC document, "Relation between Pharmaceuticals Regulatory Framework and Timely Access of Medicines to Patients: Reflection on Difficulties and Opportunities. Summary of Comments from Member States" (2014), available at http://ec.europa.eu/health//sites/health/files/files/committee/73meeting/pharm672 regulator y framework and early access annex.pdf (accessed 3 April 2017). 
highlight how the operational uncertainties surrounding the application of AP are vulnerable to exploitation and could progressively lead to the de-regulation of an already exploited direct-to-consumer market. As the EMA is currently refining the $\mathrm{AP}$ scheme with the aim to integrate the scheme into the $\mathrm{EU}$ regulatory regime, we draw attention to weaknesses in the scheme, and propose solutions to overcome these potential shortfalls.

\section{2 "Adaptive" trial methodology}

In response to calls for faster access to innovative biomedicines, advocates of more flexible regulatory pathways have proposed an alternative "adaptive" trial methodology. Under this approach, evidence is gathered and evaluated iteratively for license adaptation to reduce uncertainties rather than in an accumulative phased trial process. According to the Massachusetts Institute of Technology (MIT) Center for Biomedical Innovation:

Adaptive licensing seeks to maximize the positive impact of new drugs on public health by balancing timely access for patients with the need to access and to provide adequate evolving information on benefits and harms so that better-informed patient care decisions can be made. ${ }^{3}$

The EMA's two-year pilot project is part of a wider effort to harmonise and accelerate market access to novel therapeutics within the single market. For the pilot, the EMA selected six products out of 62 applications to trial under the AP initiative, including the one stem cell-based product, Holoclar. This product received conditional market approval under the AP scheme in December 2014 for the treatment of moderate to severe limbal stem cell deficiency caused by

H-G Eichler et al., "Adaptive Licensing: Taking the Next Step in the Evolution of Drug Approval" (2012) 91 Clinical Pharmacology E Therapeutics 426-437. 
physical or chemical burns to the eyes. Holoclar is an autologous graft of cultivated limbal stem cells and is the first stem cell-based product to be approved for marketing in the EU. Significantly, Holoclar is the only product so far approved solely on the basis of two retrospective studies in Europe. ${ }^{4}$

While many in the scientific community have welcomed this development, the regulatory approach of accelerating the approval process for novel biomedicines is in need of critical evaluation and should be viewed with some caution. The EMA is not the first regulator to adopt this approach: the Food and Drug Administration in the United States has implemented a similar scheme in the Accelerated Approval Program, which will be broadened in the newly enacted 21st Century Cures Act to include certain types of stem cells. In Japan, the Ministry for Health, Labour Welfare also introduced a regulatory framework to provide conditional approvals for regenerative medicine products.

One common concern raised with these programs is that they significantly lower the evidentiary standards needed to establish clinical efficacy and safety for highly novel biological products, thereby placing higher burdens of risk on patients without any certainty of clinical benefit. Other concerns relate to the lack of transparency in the approval process and how early market entry may discourage investment in large scale efficacy trials. Furthermore, these programs are occurring against a backdrop where private clinics and businesses are actively marketing putative stem cell-based interventions directly to patients, many for serious medical indications that lack scientific evidence that demonstrates safety and efficacy. These activities may become exacerbated in regulatory contexts that allow market authorisation of innovative medicinal products without rigorous evidence that demonstrates their safety and efficacy.

4 Giovanni Milazzo et al., "Holoclar: First of its Kind in More Ways than One" (2016) 2 Cell E Gene Therapy Insights 183-197. 


\section{Stem cell regulation under the AP scheme}

Stem cells are regulated under various legislative frameworks as either biologicals or as human cells and tissue products. ${ }^{5}$ Under the EU framework, they are regulated as Advanced Therapy Medicinal Products (ATMPs) within the centralised marketing authorisation power of the EMA. ${ }^{6}$ Currently, the EMA regulates the development and clinical translation of ATMPs within the EU under a framework of directives and regulatory guidance, which includes the Regulation on Advanced Therapy Medical Products, Regulation on Clinical Trials, Guideline on Safety and Efficacy Follow-up-risk Management of Advanced Therapy Medicinal Products, as well as relevant legislation, clinical and ethical guidelines of Member States.

Like other medicinal products available on the EU market, stem cell-based products are subject to drug labelling requirements - a summary of product characteristics providing physicians, pharmacists and other healthcare professions information on how to administer the medicine - as well as a description of the risks and ways to minimise them. However, implementation of these informational measures may vary across Member States as the requirement is enacted as a directive, meaning that the Member State must transpose the directive into national laws.

To ensure transparency of the EMA's decision-making process, sponsors of ATMPs are normally required to submit a risk management plan identifying potential risks and outlining the corresponding contingent plans as part of their marketing authorisation application. Article 14(1) of the Regulation on ATMPs

5 Tamra Lysaght et al., "Oversight for Clinical Uses of Autologous Adult Stem Cells: Lessons from International Regulations" (2013) 13 Cell Stem Cell 647-651.

6 Regulation (EC) No 1394/2007 of the European Parliament and of the Council of 13 November 2007 on Advanced Therapy Medicinal Products and amending Directive 2001/83/EC. 
states that applicants must detail "measures envisaged to ensure follow-up of efficacy of ATMPs and of adverse reactions thereof." At its discretion, the agency may impose financial sanctions if manufacturers fail to comply with the obligations stipulated in the Regulation. However, it remains to be seen if the EMA will impose sanctions on sponsors if they deviate from the risk assessment plan, as such action may elicit political backlash from the industry and deter investments in biomedical innovations. Thus, it is reasonable to expect that the EMA may be cautious about enforcing sanction.

Unlike the conventional clinical trial pathway, the AP scheme is distinguished by its regulatory flexibilities aimed at facilitating faster market access. Potential applicants for the AP scheme must first identify the relevant stakeholders, develop an iteration plan, ${ }^{7}$ and demonstrate that the collection of 'real-world data' is possible. The EMA defines real-world data as "data collected both prospectively and retrospectively from observations of routine clinical practice ${ }^{\prime \prime}{ }^{8}$ which can be gathered from various sources such as patient registries, electronic medical records, and observational studies. ${ }^{9}$ While opening up the sources of evidence that sponsors can use to support the safety and efficacy of a product, these data are also much more difficult to verify and validate.

Even though the AP uses existing regulatory approval tools, it modifies and accelerates the pathways in which clinical evidence is gathered and assessed.

7 According to the EMA, an iteration plan can either mean that the drug is approved in stages, beginning with a restricted patient population then expanded to a wider patient population or the drug confirms the benefit-risk profile, following a conditional approval based on surrogate endpoints. See EMA, "Adaptive Pathways", available at http://www.ema.europa.eu/ema/index.jsp?curl=pages/regulation/general/general content 0 00601.jsp (accessed 3 April 2017).

8 EMA, “Final Report on the Adoptive Pathway Pilot" (2016) EMA/276376/2016, available at http://www.ema.europa.eu/docs/en GB/document library/Report/2016/08/WC500211526.pd $\underline{\mathrm{f}}$ (accessed 3 April 2017).

9 Ibid. 
Significantly, the AP expands on conditional marketing approval to medicines that are likely to address an unmet medical need in a defined population, in spite of their uncertain risk-benefit profile at the time of application. Specifically, the AP scheme is markedly different from the conventional pathways by its introduction of a "life-span approach" towards clinical evaluation. This approach encompasses regulators, sponsors and stakeholders working together to decide appropriate trial designs and the selection of surrogate endpoints, as discussed further below. If the EMA issues an initial positive risk-benefit assessment, then the product is made available to a limited patient-population, which later may be expanded subject to monitoring and specific obligations.

Recognising the technical specificity inherent to ATMPs, the Regulation on ATMPs has established the Committee for Advanced Therapies (CAT), which is tasked primarily with preparing a draft opinion on the quality, safety and efficacy of each product for final approval by the Agency's Committee for Medicinal Products for Human Use (CHMP). Based on the CAT's draft report, the CHMP then submits its recommendation to the European Commission (EC). Once the EC adopts the CHMP's positive recommendation, marketing authorisation is granted and is valid throughout the internal market.

However, as the AP scheme streamlines the clinical evaluation process, it also risks lowering the clinical evaluation standard to the detriment of patient safety. We highlight two potentially problematic aspects of AP: (1) the conditional approval that is given on the basis of surrogate points rather than primary endpoints; and (2) the sponsor's capacity for long-term monitoring and compliance with post-marketing obligations. If not adequately addressed, the AP scheme may undermine patient safety, burden the market with inefficacious biomedical products, and ultimately jeopardise the EMA's reputation as a public health agency. With reference to Holoclar, the first ATMP to receive market 
authorisation from the EMA for the treatment for limbal stem cell deficiencies, the paper will now detail these two potential challenges.

\section{Potential challenges and solutions}

\subsection{Altered trial design}

The hallmark of AP is the embodiment of adaptive clinical study design, which permits changes and revisions to the assumptions of ongoing trials in light of accumulated observed data. ${ }^{10}$ This approach differs from standard clinical trials, which are designed with assumptions or hypotheses that are tested from the outset and do not allow for subsequent revision. This regulatory flexibility of the adaptive clinical trial design is appealing to both regulators and drug developers because, in theory, it increases efficiency in the allocation of clinical and regulatory resources by permitting constant revisions in trial design, facilitating faster drug development.

Although the EMA believes that conventional clinical trial design is illequipped to facilitate an efficient drug development process, ${ }^{11}$ critics remain sceptical about the suitability of adaptive clinical trial design as an alternative paradigm for stem cell-based treatments. ${ }^{12}$ Specifically, conditional market

10 Committee for Medicinal Products for Human Use, Reflection Paper on Methodological Issues in Confirmatory Clinical Trials Planned with an Adaptive Design (CHMP/EWP/2459/02, 2007), available at http://www.ema.europa.eu/docs/en GB/document library/Scientific guideline/2009/09/WC5 00003616.pdf (accessed 3 April 2017).

11 EMA, "Adaptive Pathways: a Future Approach to Bring New Medicines to Patients?", available at http://www.ema.europa.eu/ema/index.jsp?curl=pages/news and events/news/2014/12/news detail 002234.jsp\&mid=WC0b01ac058004d5c1 (accessed 3 April 2017).

12 See e.g. Alessandro Blasimme and Emmanuelle Rial-Sebbag, "Regulation of Cell-Based Therapies in Europe: Current Challenges and Emerging Issues" (2013) 22 Stem Cells and Development 14-19; Richard Scheffler (ed.), World Scientific Handbook of Global Health Economics and Public Policy: Volume 3 - Health Characteristics and Performance (Singapore: World 
approvals will be based on surrogate endpoints established in Phase II clinical studies, with post-marketing evidence replacing Phase III clinical trials. The concern is that bypassing Phase III efficacy trials may increase the risk to patients given that only around $50-60 \%$ of medicinal products pass through this phase successfully..$^{13}$

The use of surrogate, instead of clinical, endpoints is a key feature of adaptive clinical study. Surrogate endpoints are substitute biomarkers for clinical endpoints that are thought to predict clinical benefit based on epidemiological, therapeutic, or other scientific evidence. ${ }^{14}$ For Holoclar, the observation of corneal epithelial integrity and the absence of significant neovascularisation serve as proxy for primary endpoints. ${ }^{15}$ Secondary endpoints included change in symptoms (pain, burning, photophobia) from baseline to 12 months postintervention. The use of surrogate endpoints during the early drug development phase is not uncommon. The FDA, for instance, grants marketing approval for new drug products on the basis that the product has an effect on surrogate endpoint that is reasonably likely to predict clinical benefits.

Nonetheless, critics of surrogate endpoints argue that they often do not correlate with primary outcomes, such as extended quality of life years, and where they do, they may have no causal relationship with disease progression. ${ }^{16}$

Scientific Publishing, 2016); Courtney Davis et al, “'Adaptive Pathways' to Drug Authorisation: Adapting to Industry?" (2016) BMJ 354.

13 Michael Ermisch et al., "Payers' Views of the Changes Arising through the Possible Adoption of Adaptive Pathways" (2016) 7 Frontiers in Pharmacology 305.

14 Biomarkers Definition Working Group, "Biomarkers and Surrogate Endpoints: Preferred Definitions and Conceptual Framework" (2001) 69 Clinical Pharmacology Therapeutics 89-95.

15 Committee for Medicinal Products for Human Use, "Assessment Report for Holoclar" (2014) EMA/25273/2015, available at http://www.ema.europa.eu/docs/en GB/document library/EPAR Public assessment report/human/002450/WC500183405.pdf (accessed 3 April 2017).

16 Tomasz Burzykowski et al., The Evaluation of Surrogate Endpoints (1st ed., New York: Springer, 2005). 
In addition, a drug product that is approved on the basis of surrogate endpoints will add an additional level of uncertainty in assessing the product's benefit-risk profile as compared to conventional drug approval route. ${ }^{17}$ Critics also argue the AP scheme is redundant because existing regulatory mechanisms, such as compassionate use programmes, accelerated assessment and hospital exemptions, already allow expedited patient access to investigational products. ${ }^{18}$ Taken together, these objections cast doubt on whether the public health benefits of the AP scheme will outweigh the associated risks of foregoing efficacy studies with primary endpoints.

With respect to Holoclar, this product is used to treat corneal burns, which is a rare medical condition that affects 1 in 30,000 individuals. ${ }^{19}$ As the product was designated as an orphan drug previously, ${ }^{20}$ the use of surrogate endpoints may be justified due to the feasibility and time it would take to conduct largescale clinical trials on such a small patient population. However, it is unclear how the surrogate endpoints were chosen or how well they correlate with improved vision in the long-term. Since these endpoints have set the parameters for evaluating the safety and effectiveness of the product, the methodology used to determine them should be made public for independent assessment. ${ }^{21}$

Problematically, the AP scheme would in essence reverse the clinical evaluation process in which stem cell-based products are assessed. The EMA

17 Russell Katz, "Biomarkers and Surrogate Markers: An FDA Perspective" (2004) 11 NeuroRX 189-195.

18 See e.g. Davis et al., supra n. 12.

19 The EMA recommended the market approval based on retrospective case series (combined $\mathrm{n}=148)$.

20 Committee for Medicinal Products for Human Use, "Holoclar Assessment Report, EMA/25273/2015" (2015), available at http://www.ema.europa.eu/docs/en GB/document library/EPAR Public assessment report/human/002450/WC500183405.pdf (accessed 3 April 2017).

21 Insoo Hyun, Bioethics and the Future of Stem Cell Research (New York: CUP, 2013), at 172. 
argues that since the incomplete dataset at the time of conditional marketing approval would be overcome by the later data collection, only the subsequent availability of data will establish the long-term safety profile of the product. Such an argument is problematic on two key grounds. First, the argument justifies exposing patients to preventable harms on the basis of a speculative future benefit, and it overlooks the high opportunity costs for the patient and public health more broadly. Second, the EMA's argument assumes that the sponsors will voluntarily follow up with its specific obligations in collecting data after marketing approval has been granted. We elaborate the problems with postmarketing surveillance in Section 4.2.

Making the information publicly available would ensure that the decision can be verified by a third, independent and neutral party. Currently the dossier for Holoclar is not publicly available but the information can be requested. ${ }^{22}$ However, whether the information is released is subject to the EMA's discretion on a case-by-case basis. While the EMA has a legitimate concern in protecting commercially sensitive information, the process could add an additional administrative hurdle that functions as a disincentive for third party scrutiny of the chosen methodology. Equally, tracking the sponsors' follow up in their specific obligations after granting of conditional marketing is theoretically possible, but practically time consuming. One way to overcome the time constraint for data verification is to improve linkage with re-assessment reports and trial registries. Alternatively, the EU could establish a repository of postmarketing measures, similar to the one implemented by FDA, which could enhance the overall monitoring process.

22 In our experience in requesting the Holoclar dossier, the EMA was responsive to our request but the request was delayed because of the additional security clearance needed to protect sensitive commercial information contained in the dossier. 
Further, from a public health perspective, making the above information publicly accessible would help make clear the challenges to clinical application and risks associated with innovative therapies. Such knowledge would also benefit future research and refine research designs. For instance, Holoclar is approved based on its interaction with a small, defined subset of patients (two retrospective studies, with total evaluable population comprised of 135 patients according to Milazzo), ${ }^{23}$ where the CAT has publicly acknowledged that longterm uncertainties over risks remain given the nature and characteristics of stem cells. Ensuring that the information is publicly available would not only contribute to stem cell research more generally, but it would also be an important mechanism to protect patient safety. In the past, the EMA relied on information based on spontaneous reporting, pharmacoepidemiological and clinical research studies, to keep track of any significant changes in the medicine's risk-benefit profile which may warrant revocation of marketing approval. Between 2002 and 2011, for instance, the EMA withdrew 19 marketing approvals because of safety concerns, of which four were cohort studies. This again highlights the importance of transparency. ${ }^{24}$

\subsection{Post-marketing review process}

The EMA acknowledges that mandating sponsors to conduct post-marketing studies and carrying out their long-term monitoring plan would provide necessary safeguard of patient safety. However, the devil is in the detail: since the conclusion of AP pilot, the EMA has conceded that identifying

23 See supran. 3.

24 Rhian McNaughton, Gwenaël Huet, and Saad Shakir, "An Investigation into Drug Products Withdrawn from the EU Market between 2002 and 2011 for Safety Reasons and the Evidence Used to Support the Decision-Making" (2014) 4 BMJ Open e004221, available at http://bmjopen.bmj.com/content/4/1/e004221 (accessed 3 April 2017). 
methodologically sound strategies of real-world evidence collection to support the agency's initial assessment of efficacy and effectiveness remains challenging. ${ }^{25}$ Critics of AP schemes further point out that post-marketing surveillance based on observational evidence tend to overestimate the clinical benefits as compared to randomised controlled trials. ${ }^{26}$ Nonetheless, for Holoclar, the sponsor is required to submit a five-year follow-up with the final report due on June 2023, and interim reports to be provided annually during the observation period. ${ }^{27}$ According to the EMA, the sponsor of Holoclar is required to provide multinational, multicentre, prospective, open-label, uncontrolled interventional studies by December 2020. However, it is unclear from clinicaltrials.gov and the EU Clinical Trials Register whether such studies have begun. ${ }^{28}$

It is also unclear from Holoclar's risk assessment plan, whether the sponsor has the necessary financial capability to carry out the long-term monitoring activities, such as carrying out the systemic post-marketing studies beyond collecting patient health data. Given that stem cells may present risks associated with late latencies and off-target effects, ${ }^{29}$ funding and capabilitybuilding for long-term monitoring of patient's overall health status is crucialassuming sponsors and regulators follow up with the risk assessment plan. However, currently sponsors are not required to outline long-term funding and capability-funding in their risk management plan under the AP, despite

25 See supra n. 11.

26 Nigel Hawkes, "Specialists Attack Drug Agency's Fast Track Approval Scheme” (2016) 353 BMJ i3060.

27 See supra n. 15 , at 67.

28 Rita Banzi et al., "Approval of Drugs with Uncertain Benefit-Risk profiles in Europe" (2015) 26 European Journal of Internal Medicine 572-584.

29 See Hawkes, supra n. 26; see also ISSCR Guidelines on Stem Cell Research and Clinical Translation (2016), available at http://www.isscr.org/home/publications/2016-guidelines (accessed 3 April 2017). 
recommendations of the International Society for Stem Cell Research (ISSCR) Guideline 3.5.1.2 to the contrary.

Accordingly, the EMA should ensure that sponsors follow up with their post-marketing obligations to the extent possible. The EMA should also require sponsors to include plans about capability building which include long-term monitoring of patient safety and the necessary financial resources needed for monitoring as part of the risk assessment plan. Such a requirement is in keeping with the overarching goal of a risk assessment plan, which is to ensure the sponsor would take a more proactive approach toward pharmacovigilance, and minimise risks throughout a medicine's lifecycle. ${ }^{30}$

Putting aside criticisms of the shift towards a post-marketing authorisation regime, the CAT assessment report on Holoclar specifically requires the marketing-authorisation holder to "submit the first periodic safety update report... within 6 months following authorization", ${ }^{31}$ where the report is published on the European medicine web-portal in accordance with the EMA transparency policies.

The EMA should also ensure sponsors submit periodic updates on their product, and inform the EMA of any considerable adverse effects immediately. Making periodic reports publically available is an essential element of good governance. In light of the technical nature of stem cell-based therapies, the EMA should ensure that periodic reports and its decision to grant and renew conditional marketing authorisations are publicly accessible, written in plain language accessible to a layperson. As uncertainties on the therapeutic benefits of stem cell-based therapies loom large in the public sphere, making the process

30 See supra n. 24.

31 "Annex I Summary of Product Characteristics", available at http://www.ema.europa.eu/docs/en GB/document library/EPAR Product Information/human/002450/WC500183404.pdf (accessed 3 April 2017). 
transparent is one critical way to establish creditability of the stem cell industry and reaffirm the political legitimacy of the EMA in regulating the practice. Similarly, the ISSCR Guidelines affirms the obligation of the stem cell research community to provide accurate information on stem cell research. ${ }^{32}$ We further argue that such obligation extends to sponsors releasing both negative and positive results of the trial. Making these results publicly available would provide an accurate account of the trial, as well as providing additional information for future stem cell-based research.

In defense of the AP scheme, the EMA argues that if sufficient early evidence of relevant patient benefits exists to fulfil an otherwise unmet medical need, then that should justify the absence of comprehensive dataset in granting of the conditional marketing authorisation. Yet, in practice, such an approach may not only lower the evidentiary standards for granting conditional market access, but it also overlooks the fact that patient access to investigational products can be expedited using the existing regulatory mechanisms such as compassionate use without lowering standards of evidence. Institutionalising a robust and rigorous post-marketing review process may mitigate uncertainties of risks associated with stem cell-based therapies, but only to a certain extent. Critics of the AP scheme argue that once a drug is widely available, it can be difficult to remove from the market. ${ }^{33}$

The EMA further argues that the shift towards a post-marketing regime would reduce the overall cost of drug development considerably. ${ }^{34}$ While the

\footnotetext{
32 See ISSCR, supra n. 29.

33 EMA, "Adaptive Pathways Workshop. Report on a Meeting with Stakeholders Held at EMA on Thursday 8 December 2016" (2016), available at http://www.ema.europa.eu/docs/en GB/document library/Report/2017/02/WC500222153.pd $\underline{f}$ (accessed 4 April 2017).

34 See supra n. 11.
} 
EMA is of the view that the AP scheme would create regulatory certainty to mitigate financial risks for sponsors and encourage biomedicine innovations, merely lowering sponsors' financial risks in developing the drug does not mean that patients will benefit. At its worst, the scheme may reduce the financial risks for sponsors, many of those risks are likely to be shifted onto patients and public health systems who will ultimately pay for the costs of adverse events.

Likewise, the EMA's argument overlooks the high opportunity cost of the AP scheme. Specifically, as these products typically involve high clinical uncertainty over benefits and risks, the cost to patients receiving futile treatment and the cost to the public in diverting scarce public health resource cannot, and should not, be understated. Further, even assuming that sponsors would follow up with their post-marketing obligations, the AP scheme intends to rely on electronic health records and patient registries as evidence of drug efficacy. Critics and even the EMA remain doubtful over the use of observation data as indication of drug efficacy over data collected from conventional randomised clinical trials. ${ }^{35}$

Moreover, many opponents of the AP scheme have expressed doubts over the extent to which the sponsors would be able to fulfil their specific obligations once the product is granted conditional marketing approval. Such skepticism is not isolated to the critics of the AP scheme. In fact, the CHMP, in a footnote of its opinion for Holoclar acknowledges the uncertainty of sponsors to follow up, noting that "the marketing-authorization holder is likely to provide comprehensive clinical data at a later stage". ${ }^{36}$ This indicates that under the AP

35 EMA, "Adaptive Pathways Workshop", supra n. 33.

36 EMA, "Summary of Opinion. Holoclar. Ex vivo Expanded Autologous Human Corneal Epithelial cells Containing Stem Cells" (2014) EMA/CHMP/737422/2014, available at http://www.ema.europa.eu/docs/en GB/document library/Summary of opinion Initial authorisation/human/002450/WC500179301.pdf (accessed 5 April 2017). 
scheme, the obligation to provide comprehensive clinical data is not an absolute duty. Indeed, sponsors often delay fulfilling their specific obligations imposed at the time of approval. ${ }^{37}$ Despite the CHMP's scepticism on manufacturers' commitments to provide comprehensive data after post-marketing authorisation in Holoclar, the EMA remains optimistic about the AP scheme generally. Currently, it is unclear whether the EMA will resort to sanctions if the sponsor fails to comply with its specific obligation.

To strengthen the post-marketing regime, the EMA should withdraw conditional marketing approval or impose financial sanctions on manufactures who failed to follow up with their post-marketing authorisation obligations, if the manufactures cannot provide reasonable explanations for their failure to do. Both withdrawing conditional approval and imposing financial sanctions are within the EMA's legal power and mandate.

\section{Conclusion}

In many ways the AP scheme is a welcoming initiative for accelerating access to innovative technology, but such should not come at the expense of patient safety. In light of the fact that the AP scheme is still at an embryonic stage, this precludes us from drawing any firm conclusion. Nonetheless, we have identified two problematic aspects of the scheme, highlighting potential ways in which these, if unaddressed, might undermine the EU regulatory scheme and put patient safety in jeopardy. We have suggested some procedural safeguards to strengthen the AP scheme, taking into consideration the evolving science and the need for regulatory agility. That is, enclosing the methodology by which the surrogate

37 See e.g. Michael Hay et al., "Clinical Development Success rates for Investigational Drugs" (2014) 32 Nature Biotechnology 40-51. 
endpoints are decided, and strengthening the post-marketing surveillance regime where violators would face financial sanctions.

\section{Acknowledgements}

This research was supported with funding from the Humanities and Social

Science Fund of the Office of the Deputy President (Research and Training), National University of Singapore (WBS: R-171-000-055-646). 